\title{
ESTRATEGIAS DE AFRONTAMIENTO Y MALESTAR EMOCIONAL EN MUJERES CON RESULTADOS DUDOSOS DE SU MAMOGRAFÍA DE CRIBADO
}

\author{
COPING STRATEGIES AND EMOTIONAL DISTRESS IN WOMEN WITH \\ INCONCLUSIVE RESULTS IN SCREENING MAMMOGRAPHY
}

María del Carmen Cardenal Ciudad y Juan Antonio Cruzado Rodríguez

Facultad de Psicología, Universidad Complutense de Madrid.

Resumen

Objetivos: Estudiar el afrontamiento en mujeres con resultados dudosos tras una mamografía de cribado de cáncer de mama que precisan repetir la prueba a los 6 meses, y examinar el valor predictivo del afrontamiento en ansiedad y depresión en los dos momentos de medida.

Metodología: 105 mujeres (edad media $=59,9$ años, $S x=6,02$ ) con resultado BIRADS 3 en la mamografía de cribado, fueron evaluadas en afrontamiento (COPE), ansiedad y depresión (HADS), y 72 de estas mujeres fueron evaluadas 6 meses después tras una mamografía de seguimiento.

Conclusiones: Las estrategias de afrontamiento más utilizadas fueron las activas y centradas en el problema y las menos utilizadas fueron las evitativas. En el segundo momento de medida disminuyeron el uso del afrontamiento activo $(p=0,000)$, planificación $(p=0,002)$, reinterpretación positiva y crecimiento personal $(p=0,03)$ y refrenar el afrontamiento $(p=0,029)$. La ansiedad y depresión eran normales en las dos evaluaciones. Las estrategias de afrontamiento centradas en el problema son más beneficiosas que las centradas en la emoción, si bien, las correlaciones del afrontamiento con ansiedad y depresión son modestas. El cribado mamográfico es poco estresante y exige escasos recursos de afronta-
Abstract

Objetive: The aims of this research are to study the coping strategies in women with doubtful results after mammography screening for breast cancer who require repeat testing at 6 months, and analyse the predictive value of coping strategies for anxiety and depression at both times of measurement.

Methodology: One hundred and five women (mean age $=59.9$ años, $S x=6.02$ ) with BI-RADS 3 findings in a mammography screening were evaluated in coping (COPE), anxiety and depression (HADS), and 72 of these women were assessed at 6 months after a follow-up mammogram.

Conclusions: Coping strategies most used were the active and problem-focused, and the avoidant strategies were the least used. In the second measurement time decreased the use of active coping $(p=0.000)$, planning $(p=0.002)$, positive reinterpretation and growth $(p=0.03)$ and refrain coping $(p=0.029)$. Anxiety and depression were normal in both assessments. Coping strategies problem-focused are more beneficial than emotion-focused, although the correlations between coping and anxiety/depression are modest. Screening mammography does not cause distress and requires few coping resources for participants. Campaigns for breast cancer screening should emphasize these results.

Correspondencia:

Juan Antonio Cruzado Rodríguez

Facultad de Psicología

Universidad Complutense de Madrid

28223 - Madrid

E-mail: jacruzad@ucm.es 
miento para las participantes, lo cual debe ser enfatizado en las campañas de cribado.

Palabras clave: Cáncer de mama, cribado de cáncer de mama, estrategias de afrontamiento, ansiedad, depresión, malestar emocional.
Key words: Breast cancer, breast cancer screening, coping strategies, anxiety, depression, distress.

\section{INTRODUCCIÓN}

Participar en el cribado mamográfico constituye un beneficio global para las mujeres de 50 a 69 años de una reducción de alrededor del $30 \%$ de las muertes por cáncer de mama ${ }^{(1,2)}$. Probablemente conseguir un resultado normal es en un primer momento tranquilizador, pero si la mamografía es anormal, el diagnóstico puede aumentar la ansiedad ${ }^{(3-8)}$ especialmente en las mujeres a las que se les practica una biopsia, porque esta prueba se suele asociar con un problema serio de salud ${ }^{(9-11)}$. Aunque un resultado falso positivo, es decir, una mujer que tiene una mamografía con sospecha de malignidad pero en la que luego se descarta clínicamente el diagnóstico de cáncer de mama ${ }^{(12)}$ resulte finalmente un alivio, en ciertas situaciones pueden permanecer la preocupación y una alta percepción de riesgo ${ }^{(13)}$.

La mayor parte de la investigación se ha centrado en el impacto de los hallazgos falsos positivos. Sin embargo, la gran mayoría de estos estudios se han Ilevado a cabo en contextos distintos de los programas de cribado organizados ${ }^{(13)}$ : Varios autores han sugerido que el cribado de cáncer de mama podría aumentar el nivel de fobia al cáncer y el nivel de ansiedad general en la población participante ${ }^{(14-17)}$.

En una revisión de diez estudios en los que se examinó el malestar psicológico asociado con el cribado de cáncer de mama, la ansiedad aparecía como la consecuencia más prevalente de la mamografía y parecía afectar a ciertos subgrupos, siendo entre aquellas mujeres que necesi- taron mas pruebas debido a sus resultados anormales, donde se encontraron efectos más significativos ${ }^{(18)}$.

A su vez se ha encontrado que aquellas mujeres que pertenecían al grupo de las que habían recibido un resultado falso positivo, sufrían mayor ansiedad relacionada con el cáncer de mama, comparadas con aquellas mujeres que habían recibido resultados negativos, o con aquellas que nunca habían sido invitadas al cribado mamográfico, o con las que habían sido invitadas y no habían acudido ${ }^{(19)}$ y que la situación de ser llamadas para investigaciones posteriores debido a hallazgos sospechosos en la mamografía, se recordaba como una experiencia extremadamente estresante, incluso varios años después del acontecimiento ${ }^{(20)}$.

Asimismo, se ha hallado que, mujeres que en su último cribado mamográfico habían recibido un resultado claro de no malignidad después de ser evaluadas mediante una punción aspiración con aguja fina, mediante una biopsia quirúrgica o citadas a los seis meses para otra mamografía, sufrieron significativamente efectos psicológicos adversos un mes antes de volver a realizarse su mamografía de cribado, tres años después de su último resultado de no malignidad ${ }^{(21)}$.

En esta misma línea se ha encontrado que a pesar de haber recibido un resultado final de no malignidad, las mujeres que necesitaron otras investigaciones sufrieron consecuencias psicológicas adversas hasta cinco meses después de su última cita con el cribado, en mayor medida que aquellas mujeres que recibieron un resultado negativo en la mamografía ${ }^{(22)}$ y que un resultado 
falso positivo en el cribado era el predictor más importante de la ansiedad a largo plazo, dos meses después de la recepción de un resultado negativo ${ }^{(23)}$. Además, en aquellas mujeres requeridas para una revisión a corto plazo de sus resultados anormales y que finalmente no fueron diagnosticadas de cáncer de mama, los niveles de ansiedad y de depresión fueron significativamente más bajos cuatro semanas después de la realización del diagnóstico definitivo; sin embargo para las mujeres que fueron diagnosticadas de cáncer de mama, no se produjeron cambios significativos en cuanto al nivel de malestar psicológico en dicho período temporal ${ }^{(24)}$.

A este respecto, se ha encontrado, que las mujeres con resultados normales en la mamografía no vieron incrementado su malestar psicológico y disminuyó su preocupación con respecto al cáncer de mama. Sin embargo, aquellas mujeres a las que se les solicitó realizar un seguimiento de sus resultados anormales experimentaron un cierto nivel de preocupación con respecto al cáncer de mama que se mantuvo un mes después de que se confirmara el diagnóstico de benignidad ${ }^{(6)}$.

Por el contrario, varios estudios han demostrado que los efectos emocionalmente negativos son de duración breve o inexistentes: encontrando que la frecuencia de la autoexploración mamaria después del cribado solo provocaba niveles de ansiedad en una pequeña minoría de mujeres a las que se les había practicado una biopsia abierta debido a lesiones benignas. Tanto las puntuaciones de la escala de Ansiedad y Depresión Hospitalaria como las autoexploraciones mamarias mostraron que la prevalencia global de las alteraciones emocionales no se vio incrementada por el cribado mamográfico. Según este estudio, los efectos psicológicos debidos al cribado, aunque detectables, son pequeños en la población general, pero se observan en aquellos casos en los que se practica una biopsia abierta ${ }^{(25)}$.
En esta misma línea, se ha hallado que no era probable que de manera global el cribado de cáncer de mama genere efectos psicológicos adversos en las mujeres sanas, incluso cuando el resultado era un falso positivo. Atribuyéndose las diferencias en el funcionamiento psicológico entre las mujeres con un resultado falso positivo $y$ las mujeres con un resultado negativo con más probabilidad a sentimientos de alivio en el grupo cuyos resultados fueron negativos que a un aumento de la ansiedad y del malestar psicológico en el grupo al que se le dio un resultado falso positivo(26).

Comparándose mujeres que habían recibido un resultado falso positivo con mujeres que habían recibido resultados claros, tres y doce meses después de haber atendido a las pruebas posteriores se ha encontrado que no había diferencias con respecto a la ansiedad entre ambos grupos ${ }^{(27)}$.

Evaluándose el impacto psicológico de recibir un resultado falso positivo en la mamografía en el contexto de un programa poblacional de cribado de cáncer de mama, se ha encontrando que, en el momento de hacerse la mamografía de cribado las mujeres no refirieron preocupación, sin embargo, la preocupación aumentaba notablemente en el momento en que eran reconvocadas. Las mujeres que recibieron un resultado falso positivo se preocuparon más por padecer cáncer de mama que las que no pasaron por esta situación y señalaron que más información las hubiese ayudado en los momentos de mayor preocupación. Sufrir un falso positivo no afectó a la futura intención de participar en la siguiente ronda de cribado. Concluyendo que padecer un falso positivo si bien provocaba un impacto psicológico, no produjo morbilidad psicológica ${ }^{(28)}$.

El riesgo de tener cáncer de mama, la realización de pruebas de cribado y los resultados adversos de estas pruebas constituyen acontecimientos estresantes. Las estrategias de afrontamiento que utili- 
zan las mujeres en riesgo son importantes determinantes de su adaptación.

Se han encontrado diferencias entre las estrategias de afrontamiento utilizadas por mujeres que padecen cáncer de mama y aquellas estrategias de afrontamiento empleadas por mujeres que no padecen esta enfermedad. Comparando mujeres con cáncer de mama que acudieron al hospital a la realización de una revisión médica con mujeres sin patologías oncológicas que asistían de forma voluntaria al cribado se ha encontrado que las mujeres afectadas por el carcinoma mamario tenían una capacidad reducida para controlar sus emociones y tendían a usar estrategias evitativas para afrontar el estrés como la negación ${ }^{(29)}$.

Con respecto al cribado mamográfico, además de por las características de la prueba, el impacto del cribado está casi sin duda moderado por las características individuales de quienes son evaluados ${ }^{(12)}$. Algunas personas son particularmente vulnerables a la amenaza de la información. Se ha desarrollado una tipología de estilos de procesamiento de la información y se ha demostrado que las personas tienen diferentes formas de procesar la información relativa a la amenaza de la salud. Los altos incrementadores (monitors) tienden a escudriñar y amplificar las señales amenazantes, mientras que los bajos incrementadores o atenuadores (blunters) encuentran formas de distraerse de las señales amenazantes. El estilo incrementador puede dar lugar a reacciones de ansiedad debido a la atención a información relativa a la amenaza de la salud ${ }^{30-32}$.

Son varios los estudios que en la actualidad han revisado la relación entre los estilos de afrontamiento y el ajuste emocional en el contexto del cribado de cáncer de mama. Encontrándose en mujeres que habían sido sometidas a una biopsia después de recibir resultados anormales en el cribado mamográfico, que un uso frecuente de la confrontación (expresión de las emo- ciones, y reestructuración cognitiva) estuvo asociado con una mayor adaptación ${ }^{(33)}$. Por el contrario, se ha puesto de manifiesto que un uso mayor de la evitación como estrategia de afrontamiento para superar la situación de volver a ser citadas para una evaluación de seguimiento, estuvo asociada con un mejor ajuste emocional en dicho seguimiento ${ }^{(34)}$. Finalmente, en consonancia con éstos hallazgos, se examinaron los niveles presentes y posteriores de malestar emocional asociados a la mamografía de cribado y la relación entre el afrontamiento de dichas mujeres y su ajuste psicosocial, encontrando que la evitación cognitiva juega un papel importante en la reducción de la ansiedad en las mujeres a las que se las vuelve a requerir para clarificar un procedimiento de cribado ambiguo(35).

En esta misma línea se ha estudiado la relación entre los estilos de afrontamiento y el ajuste emocional de las mujeres con un riesgo elevado de padecer cáncer de mama. Evaluándose las consecuencias psicológicas de una vigilancia regular para prevenir el cáncer de mama en mujeres con mayor riesgo hereditario de padecer la enfermedad, se ha encontrando que la búsqueda de apoyo social, la expresión de las emociones y los pensamientos reconfortantes están asociados con menores niveles de malestar psicológico ${ }^{(36)}$. Por el contrario el afrontamiento pasivo esta asociado tanto con niveles de malestar psicológico general como con niveles de malestar psicológico específico relacionado con el cáncer de mama. A este respecto, se examinaron las diferencias con respecto a la edad en el control emocional y sus consecuencias en mujeres requeridas para hacerse una mamografía bajo sospecha de cáncer de mama pero con resultados benignos en dicho examen médico hallando un mayor control emocional en las mujeres mayores (de entre 60 y 85 años). Esto indica que en este grupo de edad, el afrontamiento focalizado en la emoción es más corriente que 
en mujeres más jóvenes, incluso cuando ambas experimentan estresores similares. Los análisis revelaron una interacción entre la edad y el control emocional; durante el curso del estudio, en el grupo de las mujeres más mayores se relacionaron altos niveles de control con una reducción del malestar psicológico mientras que el control emocional no estuvo relacionado con cambios en el malestar psicológico ni en el grupo de las mujeres más jóvenes (de entre 19 y 39 años), ni en el grupo de las mujeres de mediana edad (de entre 40 y 59 años) ${ }^{(37)}$.

\section{Objetivos de la investigación}

Esta investigación es un estudio longitudinal cuyo objetivo general es determinar cuáles son las estrategias de afrontamiento más frecuentemente utilizadas por las mujeres que están Ilevando a cabo pruebas de cribado mamográfico, y estudiar el valor predictivo de dichas estrategias en cuanto a los niveles de ansiedad y depresión en los dos momentos de medida.

\section{MATERIAL Y MÉTODOS}

\section{Población de referencia}

Mujeres residentes en la Comunidad de Madrid, con edades comprendidas entre 50-69 años cuya mamografía ha sido catalogada como BI-RADS 3, es decir el resultado de dicha exploración es probablemente benigno, pero requiere seguimiento a corto plazo (seis meses después de la realización de la mamografía de cribado).

\section{Procedimiento}

\section{Fase I: Captación de las participantes en el estudio}

En el período comprendido desde septiembre de 2007 hasta noviembre de 2009, se contactó por vía telefónica desde la Oficina Regional de Coordinación Oncológica (ORCO) con 141 las mujeres de 50-69 años, a las que se les realizó una mamografía de cribado en el año 2007 o en el año 2008, siendo dicha mamografía catalogada posteriormente como BI-RADS 3. De las 141 mujeres, 114 (80,85\%) pertenecían al distrito de Alcorcón y 27 pertenecían al distrito de Móstoles (19,15\%). De las 114 mujeres contactadas del distritito de Alcorcón, rehusaron participar en el estudio 34 (29,82\%) y de las 27 mujeres del distrito de Móstoles, 2 (7,41\%) no quisieron participar en el mismo. La muestra final fue de 105 mujeres $^{(38)}$.

\section{Fase II: Evaluación de las participantes}

La toma de contacto con las mujeres que son objeto de nuestro estudio se realizó desde la Oficina Regional de Coordinación Oncológica, utilizando para ello los datos de las mujeres que participan en el Programa de cribado. Antes de comenzar con su evaluación se solicitó expresamente su consentimiento para participar en la investigación.

Las participantes en el estudio fueron evaluadas en dos momentos de medida. En ambos momentos la evaluación se llevó a cabo mediante entrevista telefónica:

1. Momento de medida 1: Se realizó una primera entrevista una vez que a las participantes les habían realizado la mamografía de cribado y conocían su resultado de seguimiento a corto plazo (BI-RADS 3). En el momento de esta entrevista todavía no habían acudido a realizarse la mamografía de seguimiento. Esta primera evaluación telefónica se produjo una media de 2,56 meses después del envío de la carta en la que se comunicaba el resultado.

2. Momento de medida 2: La segunda y última entrevista telefónica se Ilevó a cabo una vez que a las partici- 
pantes se les había realizado la mamografía de seguimiento y conocían que su resultado era negativo. Esta última evaluación se produjo con una media de 10,47 meses después del envió del resultado negativo.

\section{Instrumentos de medida}

Escala de Ansiedad y Depresión Hospitalaria $(\boldsymbol{H A D S})^{(39)}$ : Es un cuestionario autoaplicado de 14 ítems, integrado por dos subescalas de 7 ítems, una de ansiedad (ítems impares) y otra de depresión (ítems pares). La intensidad o frecuencia del síntoma se evalúa en una escala de Likert de 4 puntos (rango 0-3), con diferentes formulaciones de respuesta. La puntuación final de las dos subescalas se obtiene sumando las de los ítems que las componen. El marco temporal, aún cuando las preguntas están planteadas en el presente, debe referirse a la semana previa. Los criterios establecidos por Snaith para incluir o excluir a un enfermo del grupo de los que padecen trastornos emocionales son: 0-7 ausente de síntomas; 8-10 caso dudoso; $\geq 11$ presenta síntomas de depresión y/o ansiedad.

La escala ha sido traducida y validada al castellano. El coeficiente alfa de Cronbach de la escala es de 0,80 para cada una de las subescalas ${ }^{(40)}$. La validez concurrente se analizó mediante el cálculo de la correlación de Pearson entre la dimensión de Ansiedad del HADS y el STAI y la dimensión de Depresión y el BDI, obteniéndose en ambos casos valores de $0,70^{(41)}$.

$\boldsymbol{C O P E}^{42)}$ : Evalúa estrategias de afrontamiento ante el estrés. Está basado en el modelo de estrés de Lazarus y en el modelo de autorregulación propuesto por los propios autores de la escala. Esta formada por 60 ítems que describen distintos modos de afrontamiento. Puede aplicarse de dos maneras: a) pidiéndole a la persona que señale el modo en que habitualmente se enfrenta a las situaciones estresantes (ver- sión disposicional), o b) referida a alguna situación concreta (versión específica). La escala evalúa los siguientes modos de afrontamiento: afrontamiento activo, planificación, búsqueda de apoyo instrumental, búsqueda de apoyo social, supresión de actividades distractoras, religión, reinterpretación positiva y crecimiento personal, refrenar el afrontamiento, aceptación, centrarse en emociones y desahogarse, negación, desconexión mental y desconexión conductual. Asimismo incluye ítems que evalúan otros dos modos de afrontamiento (consumo de alcohol o drogas y humor). Para registrar las respuestas de las personas utiliza una escala tipo Likert de 4 puntos, donde 1 significa "habitualmente no hago esto en absoluto" y 4 "habitualmente hago esto mucho". Se puede obtener una puntuación para cada uno de estos modos de afrontamiento o una puntuación total, cuyo rango varía entre 60 y 240. El tiempo de aplicación es de 15-20 minutos ${ }^{(43)}$. En nuestro trabajo hemos optado por obtener una puntuación para cada uno de los modos de afrontamiento. Propiedades psicométricas: Fiabilidad: consistencia interna: 0,45-0,92. Fiabilidad test-retest: 0,46-0,86 (8 semanas) y 0,42-0,89 (6 semanas). Correlación versión general y situacional: menor de 0,40 (salvo escalas de consumo de alcohol o drogas y religión).Validez: Muestra relaciones con otras escalas de afrontamiento y medidas de personalidad. Así, existe una relación positiva entre las estrategias de afrontamiento activo, planificación, reinterpretación positiva y crecimiento personal con otras variables como optimismo, control de la situación, autoestima y dureza (hardiness). El afrontamiento activo presenta además una relación positiva con la personalidad tipo A, y una relación negativa con ansiedad-rasgo. Las estrategias de negación y desconexión conductual presentan un patrón opuesto en sus relaciones con estas variables. Por otra parte, las escalas del COPE se diferencian de otras 
medidas de afrontamiento como la medida unidimensional del atenuador (blunting) vs. incrementador (monitoring), y no están relacionadas con la deseabilidad social ${ }^{(43)}$.

En el momento de medida 1 se pidió a las participantes que pensaran en el acontecimiento más estresante que les había sucedido en los últimos meses. Sus respuestas se agruparon en: "acontecimiento estresante no relacionado con la mamografía de cribado"; "la espera de los resultados y/o la realización de la mamografía de cribado es el acontecimiento más estresante"; "la espera de los resultados y/o la realización de la mamografía es nombrada de forma secundaria" y "no le ha sucedido ningún acontecimiento estresante".

En el momento de medida 2, se volvió a pedir a las participantes que pensaran en el acontecimiento más estresante que les había sucedido en los últimos meses. Sus respuestas se agruparon en: "acontecimiento estresante no relacionado con la segunda mamografía a los seis meses"; "la espera de los resultados y/o la realización de la segunda mamografía a los seis meses es el acontecimiento más estresante"; "la espera de los resultados y/o la realización de la segunda mamografía a los seis meses es nombrada de forma secundaria" y "no le ha sucedido ningún acontecimiento estresante".

\section{RESULTADOS}

\section{Características sociodemográficas de las mujeres participantes}

La media de edad de las 105 participantes fue de 59,93 años $(S x=6,02)$ con un rango entre 49,64 años y 73,13 años. La mayoría de estas mujeres el $76 \%(N=80)$ estaban casadas y el $50,5 \%(N=53)$ de su totalidad completaron los estudios primarios. De las 105 mujeres que dieron su consentimiento para participar en el estudio, 33 no hicieron la segunda entrevista, por lo que finalmente se obtuvo una muestra de 72 participantes para la realización de los análisis bivariados ${ }^{(38)}$. Los motivos por los cuales éstas 33 mujeres no fueron entrevistadas en el segundo momento de medida se encuentran recogidos en la tabla 1.

\section{Estado emocional}

Los niveles de ansiedad y depresión evaluados con el HADS son bajos en ambos momentos de medida y no difieren de la media del baremo para la población general. La mayor parte de las participantes estuvieron en el rango de la normalidad, y puntuaron dentro de lo calificado en el HADS como niveles normales de ansiedad y depresión en ambos momentos de medida, sin que se produjeran cambios entre ellos ${ }^{(38)}$.

\section{Afrontamiento}

El $55,2 \%$ de las participantes $(N=58)$ expresó un acontecimiento estresante sucedido en los últimos meses no relacionado con la mamografía de cribado, el 20\% $(N=21)$ de las participantes expresó que no le había sucedido ningún acontecimiento estresante, el 15,2\% ( $N=16)$ nombró la realización de la mamografía y/o la espera de los resultados de forma secundaria y para el $9,5 \%(N=10)$ de las mujeres entre-

\section{Tabla 1. Motivo de no entrevista después de la mamografía a los 6 meses}

\begin{tabular}{|c|c|c|c|c|c|}
\hline $\begin{array}{c}\text { No se hace la } \\
\text { mamografía por } \\
\text { el DEPRECAM }\end{array}$ & $\begin{array}{c}\text { No } \\
\text { localizada }\end{array}$ & $\begin{array}{c}\text { Necesitan hacerse } \\
\text { más pruebas } \\
\text { complementarias }\end{array}$ & $\begin{array}{c}\text { No le interesa } \\
\text { hacer la segunda } \\
\text { entrevista }\end{array}$ & $\begin{array}{c}\text { Datos } \\
\text { duplicados }\end{array}$ & Total \\
\hline $5(15,2 \%)$ & $12(36,4 \%)$ & $12(36,4 \%)$ & $3(9,1 \%)$ & $1(3,0 \%)$ & $33(100 \%)$ \\
\hline
\end{tabular}


vistadas la espera de los resultados y/o la realización de la mamografía de cribado fue el acontecimiento más estresante vivido en los últimos meses.

El $56,9 \%(N=41)$ de las participantes comentaron acontecimientos estresantes no relacionados con la segunda mamografía a los seis meses y el $43,1 \% \quad(N=31)$ expresó no haber vivido acontecimiento estresante alguno en los últimos meses.

En la tabla 2 se muestran los estadísticos descriptivos de las medidas de afrontamiento en el primer momento de medida de la totalidad de las participantes $(\mathrm{N}=105)$ y en la tabla 3 los estadísticos descriptivos de las medidas de afrontamiento en ambos momentos de medida de las 72 participantes que realizaron ambas evaluaciones.

En el momento de medida 1, cabe destacar que las estrategias que han uti- lizado son fundamentalmente de tipo activo y centradas en el problema. Las más predominantes son: reinterpretación positiva y crecimiento personal, afrontamiento activo y planificación, seguidas de religión, aceptación y apoyo social. En contraste las menos utilizadas son las estrategias evitativas: consumo de drogas, desconexión conductual y negación. Cabe suponer que frente a una situación potencialmente estresante como el cribado mamográfico que tiene características de controlabilidad las estrategias más adaptativas deberían ser las activas, que son las que utilizan la mayor parte de las mujeres que participan.

En el momento de medida 2, las estrategias más predominantes son: reinterpretación positiva y crecimiento personal, religión, planificación, aceptación y afron-

\section{Tabla 2. Descriptivos de la variable afrontamiento en el primer momento de medida para la totalidad de las participantes que llevaron a cabo la primera evaluación.}

\begin{tabular}{|c|c|c|c|c|c|}
\hline \multicolumn{5}{|c|}{ Afrontamiento en el momento $1(\mathrm{~N}=105)$} \\
\hline & $\begin{array}{c}\text { Mínimo- } \\
\text { Máximo }\end{array}$ & $M$ & $D T$ & \multicolumn{2}{|c|}{$\begin{array}{c}\text { Intervalo de confianza } \\
\text { de la media al 95\% }\end{array}$} \\
\hline Afrontamiento activo & $4-16$ & 14,14 & 2,95 & 13,57 & 14,71 \\
\hline Planificación & $4-16$ & 13,50 & 3,32 & 12,85 & 14,14 \\
\hline Apoyo instrumental & $4-16$ & 11,33 & 4,60 & 10,44 & 12,22 \\
\hline Apoyo social & $4-16$ & 12,10 & 4,40 & 11,25 & 12,96 \\
\hline Supresión de actividades distractoras & $4-16$ & 9,72 & 3,53 & 9,04 & 10,41 \\
\hline Religión & $4-16$ & 12,68 & 3,49 & 12,00 & 13,35 \\
\hline Reinterpretación positiva y crecimiento & $4-16$ & 13,97 & 3,31 & 13,33 & 14,61 \\
\hline personal & $4-16$ & 11,64 & 4,26 & 10,81 & 12,46 \\
\hline Refrenar el afrontamiento & $5-16$ & 12,87 & 2,80 & 12,32 & 13,41 \\
\hline Aceptación & $4-16$ & 8,55 & 4,39 & 7,70 & 9,40 \\
\hline Centrarse en emociones y desahogarse & $4-16$ & 6,64 & 2,88 & 6,08 & 7,19 \\
\hline Negación & $4-14$ & 8,68 & 2,32 & 8,23 & 9,12 \\
\hline Desconexión mental & $4-14$ & 6,33 & 2,73 & 5,80 & 6,86 \\
\hline Desconexión conductual & $4-4$ & 4,00 & 0,00 & & \\
\hline Consumo de drogas & $4-16$ & 7,19 & 3,67 & 6,48 & 7,90 \\
\hline Humor & & & & \\
\hline
\end{tabular}




\section{Tabla 3. Descriptivos de la variable afrontamiento en ambos momentos de medida para las participantes que completaron ambas evaluaciones.}

\begin{tabular}{|c|c|c|c|c|c|c|c|c|c|c|}
\hline \multicolumn{6}{|c|}{ Afrontamiento en el momento $1(\mathrm{~N}=72)$} & \multicolumn{5}{|c|}{$\begin{array}{l}\text { Afrontamiento en el momento } 2 \\
\qquad(N=72)\end{array}$} \\
\hline & $\begin{array}{l}\text { Mínimo- } \\
\text { Máximo }\end{array}$ & M & $D T$ & $\begin{array}{r}\text { Interv } \\
\text { confia } \\
\text { la me } \\
9\end{array}$ & $\begin{array}{l}\text { alo de } \\
\text { רza de } \\
\text { dia al } \\
\%\end{array}$ & $\begin{array}{l}\text { Mínimo- } \\
\text { Máximo }\end{array}$ & M & $D T$ & $\begin{array}{r}\text { Interv } \\
\text { confia } \\
\text { la me } \\
95\end{array}$ & $\begin{array}{l}\text { lo de } \\
\text { hza de } \\
\text { dia al } \\
\%\end{array}$ \\
\hline $\begin{array}{l}\text { Afrontamiento } \\
\text { activo }\end{array}$ & $4-16$ & 14,17 & 3,06 & 13,45 & 14,89 & $6-16$ & 11,64 & 1,77 & 11,22 & 12,05 \\
\hline Planificación & $4-16$ & 13,29 & 3,50 & 12,47 & 14,11 & $5-16$ & 12,57 & 2,81 & 11,91 & 13,23 \\
\hline $\begin{array}{c}\text { Apoyo } \\
\text { instrumental }\end{array}$ & $4-16$ & 11,96 & 4,53 & 10,89 & 13,02 & $4-16$ & 11,15 & 3,60 & 10,31 & 12,00 \\
\hline Apoyo social & $4-16$ & 12,46 & 4,41 & 11,42 & 13,49 & $4-16$ & 12,26 & 3,68 & 11,40 & 13,13 \\
\hline $\begin{array}{c}\text { Supresión de } \\
\text { actividades } \\
\text { distractoras }\end{array}$ & $4-16$ & 9,86 & 3,59 & 9,02 & 10,70 & $5-16$ & 9,88 & 3,07 & 9,15 & 10,60 \\
\hline Religión & $4-16$ & 12,65 & 3,52 & 11,83 & 13,48 & $4-16$ & 12,78 & 3,32 & 12,00 & 13,56 \\
\hline $\begin{array}{c}\text { Reinterpretación } \\
\text { positiva y } \\
\text { crecimiento } \\
\text { personal }\end{array}$ & $4-16$ & 14,19 & 3,24 & 13,43 & 14,96 & $7-16$ & 13,71 & 2,39 & 13,15 & 14,27 \\
\hline $\begin{array}{c}\text { Refrenar el } \\
\text { afrontamiento }\end{array}$ & $4-16$ & 11,67 & 4,35 & 10,65 & 12,69 & $4-16$ & 10,72 & 3,60 & 9,88 & 11,57 \\
\hline Aceptación & $5-16$ & 12,61 & 2,97 & 11,91 & 13,31 & $7-16$ & 12,22 & 2,32 & 11,68 & 12,77 \\
\hline $\begin{array}{l}\text { Centrarse en } \\
\text { emociones y } \\
\text { desahogarse }\end{array}$ & $4-16$ & 8,83 & 4,54 & 7,77 & 9,90 & $4-16$ & 8,89 & 4,07 & 7,93 & 9,84 \\
\hline Negación & $4-16$ & 6,90 & 3,07 & 6,18 & 7,62 & $4-16$ & 6,44 & 2,64 & 5,82 & 7,06 \\
\hline $\begin{array}{l}\text { Desconexión } \\
\text { mental }\end{array}$ & $4-14$ & 8,82 & 2,33 & 8,27 & 9,37 & $4-15$ & 9,25 & 2,51 & 8,66 & 9,84 \\
\hline $\begin{array}{l}\text { Desconexión } \\
\text { conductual }\end{array}$ & $4-14$ & 6,40 & 2,65 & 5,78 & 7,03 & $4-11$ & 5,99 & 2,02 & 5,51 & 6,46 \\
\hline $\begin{array}{l}\text { Consumo de } \\
\text { drogas }\end{array}$ & $4-4$ & 4,00 & 0,00 & & & $4-4$ & 4,00 & 0,00 & & \\
\hline Humor & $4-16$ & 7,54 & 3,86 & 6,64 & 8,45 & $4-16$ & 7,00 & 3,38 & 6,21 & 7,79 \\
\hline
\end{tabular}


tamiento activo y las estrategias evitativas siguen siendo minoritarias. De modo que las estrategias de afrontamiento activas siguen manteniéndose, pero dado que el hecho estresante ha desaparecido se centran más en encontrar un beneficio de la experiencia del cribado.

Únicamente se han encontrado diferencias significativas entre ambos momentos de medida en las subescalas: Afrontamiento activo; Planificación; Reinterpretación positiva y crecimiento personal y Refrenar el afrontamiento.

En la tabla 4 se muestran las diferencias entre ambos momentos de medida en las subescalas de afrontamiento con resultados significativos

La disminución encontrada en el afrontamiento activo entre el momento 1 y el momento 2 es importante dado que el tamaño del efecto es de 1,428, lo que sugiere que quizás si en el primer momento las mujeres se han enfrentado al riesgo maximizando una estrategia activa, en el segundo momento una vez que ya han realizado las pruebas y conocen que su resultado es negativo hay una menor necesidad del afrontamiento activo.

\section{Ansiedad y afrontamiento}

\section{Ansiedad en el momento 1}

Los resultados muestran que se da una correlación negativa entre la ansiedad en el momento uno y la reinterpretación positiva de -0,293; $p=0,002$. Así como una correlación positiva entre ansiedad y centrarse en emociones y desahogarse de 0,225; $p=0,021$. En ambos casos son relaciones pequeñas en las que la variable ansiedad explica respectivamente el $8,5 \%$ y el $5 \%$ de la varianza de las subescalas de afrontamiento respectivas.

\section{Tabla 4. Diferencias entre el momento 1 y el momento 2 en las subescalas de afrontamiento y la comprobación de su significación mediante la prueba de Wilcoxon.}

\begin{tabular}{|c|c|c|c|c|c|c|}
\hline & $M_{e}$ & $\begin{array}{l}\text { Diferencias } \\
\text { de medias }\end{array}$ & Z & $p$ & $\begin{array}{c}\text { Tamaño } \\
\text { del } \\
\text { efecto }\end{array}$ & Conclusión \\
\hline Afrontamiento activo 1 & 16 & \multirow{2}{*}{4} & \multirow{2}{*}{$-5,65$} & \multirow{2}{*}{0,000} & \multirow{2}{*}{1,428} & \multirow{2}{*}{$\begin{array}{c}\text { Disminuye en el } \\
\text { momento } 2\end{array}$} \\
\hline Afrontamiento activo 2 & 12 & & & & & \\
\hline Planificación 1 & 14,5 & \multirow[b]{2}{*}{1,5} & \multirow[b]{2}{*}{$-3,14$} & \multirow[b]{2}{*}{0,002} & \multirow[b]{2}{*}{0,257} & \multirow{2}{*}{$\begin{array}{l}\text { Disminuye en el } \\
\text { momento } 2\end{array}$} \\
\hline $\begin{array}{l}\text { Planificación después de la } \\
\text { mamografía a los } 6 \text { meses }\end{array}$ & 13 & & & & & \\
\hline $\begin{array}{l}\text { Reinterpretación positiva y } \\
\text { crecimiento personal } 1\end{array}$ & 16 & \multirow{2}{*}{1,5} & \multirow{2}{*}{$-2,17$} & \multirow{2}{*}{0,03} & \multirow{2}{*}{0,204} & \multirow{2}{*}{$\begin{array}{l}\text { Disminuye en el } \\
\text { momento } 2\end{array}$} \\
\hline $\begin{array}{l}\text { Reinterpretación positiva y } \\
\text { crecimiento personal } 2\end{array}$ & 14,5 & & & & & \\
\hline Refrenar el afrontamiento 1 & 16 & \multirow{2}{*}{5,5} & \multirow{2}{*}{$-2,18$} & \multirow{2}{*}{0,029} & \multirow{2}{*}{0,262} & \multirow{2}{*}{$\begin{array}{l}\text { Disminuye en el } \\
\text { momento } 2\end{array}$} \\
\hline Refrenar el afrontamiento 2 & 10,5 & & & & & \\
\hline
\end{tabular}


Ansiedad en el momento 2

Se encontraron correlaciones significativas de Pearson entre la variable ansiedad y "apoyo instrumental" $(r=0,27, p=0,024$; varianza explicada $7 \%$ ), y entre la variable ansiedad y "supresión de las actividades distractoras" ( $r=0,33, p=0,005$; varianza explicada 11\%). Asimismo, se encontró una correlación significativa mediante el estadístico rho de Spearman entre la variable ansiedad y la subescala de afrontamiento "centrarse en emociones y desahogarse" (rho $=0,255, \mathrm{p}=0,031$; varianza explicada $65 \%$ ).

\section{Depresión y afrontamiento}

\section{Depresión en el momento 1}

Como era de esperar, los resultados muestran que existe una correlación significativa entre la variable depresión y la subescala de afrontamiento "centrarse en emociones y desahogarse" (rho $=0,26$, $p=0,007$; varianza explicada $=6,7 \%$ ) y entre depresión y la subescala de afrontamiento "humor" (rho=-0,204, p=0,037; varianza explicada $=4 \%$ ).

La capacidad predictiva de la depresión con respecto a las estrategias de afrontamiento es muy escasa.

\section{Depresión en el momento 2}

Los resultados mostraron que solamente se dio una correlación significativa entre la depresión y la subescala de afrontamiento "humor" (rho=-0,234, p=0,048; varianza explicada $=5,5 \%$ ). Los datos confirman de nuevo la baja capacidad predictiva de la depresión sobre las estrategias de afrontamiento.

\section{DISCUSIÓN}

En el primer momento de medida, las estrategias de afrontamiento más utiliza- das por las mujeres participantes son de tipo activo y centradas en el problema y las menos utilizadas son las estrategias de afrontamiento de tipo evitativo. En el segundo momento, las estrategias de afrontamiento activas se mantienen pero puesto que el suceso potencialmente estresante ha desaparecido, las participantes se centran en los aspectos beneficiosos de la experiencia del cribado mamográfico. Las estrategias menos utilizadas siguen siendo las de tipo evitativo.

Con respecto al cambio de las subescalas de esta variable entre ambos momentos de medida, solo se han apreciado diferencias significativas entre el momento 1 y el momento 2 en las subescalas de: afrontamiento activo, planificación, reinterpretación positiva y crecimiento personal y refrenar el afrontamiento, produciéndose en todas ellas una disminución de la puntuación en el segundo momento de medida, que es en todas ellas de escasa significación clínica, exceptuando en la subescala afrontamiento activo. Esta disminución en el afrontamiento activo puede deberse a que en el segundo momento de medida cuando las participantes ya conocían que sus resultados eran negativos no tenían necesidad de seguir manteniendo esta estrategia de afrontamiento.

La capacidad predictiva de la variable ansiedad con respecto a las estrategias de afrontamiento es reducida. En el momento de medida 1, únicamente existieron correlaciones significativas entre la variable ansiedad y las estrategias de afrontamiento reinterpretación positiva y crecimiento personal, y centrarse en emociones $y$ desahogarse. Asimismo, en el momento 2 las correlaciones significativas encontradas fueron entre la variable ansiedad y las estrategias de afrontamiento apoyo instrumental, supresión de actividades distractoras y centrarse en emociones y desahogarse. 
La capacidad predictiva de la depresión en relación a las estrategias de afrontamiento es todavía más escasa que la de la variable ansiedad. En el momento de medida 1 únicamente han existido correlaciones significativas con las estrategias de afrontamiento centrarse en emociones $y$ desahogarse, y humor. En el momento de medida 2 la única correlación significativa ha sido con la estrategia de afrontamiento humor.

El valor predictivo del afrontamiento con respecto a los síntomas de ansiedad y depresión es muy reducido, en contraste con otros trabajos ${ }^{(34)}$ que encontraron que un uso mayor de la evitación como estrategia de afrontamiento para superar la situación de volver a ser citadas para una evaluación de seguimiento, estuvo asociada con una mejor adaptación emocional en dicho seguimiento, si bien también informan que el afrontamiento activo es un amortiguador del estrés. Asimismo, se ha mostrado que la evitación cognitiva juega un papel importante en la reducción de la ansiedad en las mujeres a las que se las vuelve a requerir para clarificar un procedimiento de cribado ambiguo ${ }^{(35)}$. Pero en el caso de las participantes de nuestro estudio, el carácter preventivo del cribado, pese a que pasar por cada una de sus fases pudiera ser potencialmente estresante, hace que las estrategias más adaptativas sean las de tipo activo tanto para afrontar el paso por las fases del cribado por las que pasarían la totalidad de las mujeres, como para afrontar la realización de una nueva mamografía a los seis meses para clarificar el diagnóstico y la espera del resultado de las misma. En el estudio referenciado anteriormente, los niveles de ansiedad de las mujeres eran altos ${ }^{(35)}$, cosa que no sucede en el presente estudio donde los niveles son bajos, por otro lado en el estudio presente la mamografía no era un estresor especialmente importante, a diferencia de esos estudios.
Por lo tanto, el hecho de que las participantes experimenten bajos niveles de ansiedad y depresión unido a que no identifiquen como acontecimientos estresantes ni la realización de las mamografías ni la recepción de los resultados de las mismas, indica que el procedimiento de cribado mamográfico es poco estresante, nada perturbador y por lo tanto no exige que las mujeres que toman parte en este programa tengan que disponer de recursos de afrontamiento para enfrentarse a la situación de cribado, incluso si experimentan, como en el caso de las mujeres sujeto de nuestro estudio, el tener que realizarse una nueva mamografía para clarificar un posible diagnóstico de benignidad. Esa es la razón principal de la ausencia de correlaciones entre las estrategias de afrontamiento con la ansiedad y depresión.

Asimismo, las campañas de cribado mamográfico deben enfatizar que la realización de la mamografía en el Programa de Detección Precoz de Cáncer de Mama de la Comunidad de Madrid (DEPRECAM) no genera malestar psicológico y por lo tanto, animar a las mujeres a que utilicen esta herramienta de prevención, así como enfatizar y concienciar a las mujeres en el uso de estrategias de afrontamiento centradas en el problema que, como se ha visto en este trabajo, en la situación de tener que clarificar un diagnóstico de benignidad, resultan más útiles que las estrategias de afrontamiento centradas en la emoción.

\section{Limitaciones del estudio}

La forma ideal de realizar el presente estudio habría sido evaluar a las mujeres a medida que pasan por todas las fases posibles del Programa, es decir, antes de hacerse la mamografía, una vez que se la han hecho pero antes de conocer el resultado de la misma, una vez que cono- 
cen el resultado de seguimiento a corto plazo, después de la realización de la mamografía de seguimiento y finalmente cuando conocen que su resultado es negativo. Pero debido a la falta de medios humanos para llevar a cabo estas evaluaciones, se optó por evaluarlas, una primera vez, a la espera de la realización de la mamografía de seguimiento a corto plazo (mamografía realizada a los seis meses de la mamografía de cribado para clarificar el diagnóstico) y una segunda vez, cuando conocen que el resultado de su mamografía de seguimiento a corto plazo es negativa.

Asimismo, el tamaño reducido de la muestra impide alcanzar una potencia estadística óptima para establecer asociaciones significativas entre las variables de nuestro estudio.

Finalmente, los meses que han pasado entre la recepción de los resultados y la valoración, especialmente en la segunda medida, son también una limitación propiciada por la falta de personal a la hora de realizar las entrevistas.

\section{Agradecimientos}

A la Oficina Regional de Coordinación Oncológica de la Comunidad Autónoma de Madrid por poner a nuestro alcance la infraestructura necesaria para la realización de este trabajo de investigación. En especial a Andrés González, a María José González y a Vicente Valentín. A Ángel Rodríguez por sus aportaciones a la fase de diseño del estudio. A Jesús Martínez, por su inestimable ayuda y asesoramiento en la parte estadística de éste trabajo. Y muy especialmente, a todas las mujeres de Alcorcón y Móstoles que dieron algún tipo de información para que esta investigación fuera posible; gracias por la colaboración recibida y por la paciencia, la generosidad y la honestidad mostradas.

\section{REFERENCIAS BIBLIOGRÁFICAS}

1. Shapiro S, Venet W, Strax P, Venet L. Periodic screening for breast cancer. The Health Insurance Plan Project and its sequeale 1963-1986. Baltimore: The Johns Hopkins University Press, 1988.

2. Tabár L, Fagerberg CJ, Gad A, Baldetorp L, Holmberg LH, Gröntoft O, et al. Reduction in mortality from breast cancer after mass screening with mammography. Randomised trial from the Breast Cancer Screening Working Group of the Swedish National Board of Health and Welfare. Lancet 1985:1(8433):829-32. Doi: 10.1016/S0140-6736(85)92204-4

3. Aro AR, Pilvikki Absetz S, van Elderen TM, van der Ploeg E, van der Kamp LJ. False-positive findings in mammography screening induces short-term distressbreast cancer specific concern prevails longer. Eur J Cancer 2000;36:1089-97. Doi: 10.1016/S0959-8049(00)00065-4

4. Brett J, Bankhead C, Henderson B, Watson E, Austoker J. The psychological impact of mammographic screening. A systematic review. Psychooncology 2005;14:917-38. Doi: 10.1002/pon.904

5. Fridfinnsdottir EB. Icelandic women's identification of stressors and social support during the diagnostic phase of breast cancer. J Adv Nurs1997;25:526-31. Doi: 10.1046/ j.1365-2648.1997.t01-1-1997025526.x

6. Lowe JB, Balanda KP, Del Mar C, Hawes E. Psychological distress in women with abnormal findings in mass mammography screening. Cancer 1999;85:1114-8. Doi: 10.1002/(SICI)10970142 (19990301)85:5<1114::AID CNCR15>3.0.CO;2-Y

7. Ong G, Austoker J. Recalling women for further investigation of breast screening: Women's experiences at the clinic and afterwards. J Public Health Med 1997;19:29-36.

8. Sandín B, Chorot P, Valiente RM, Lostao L, Santed MA. Anticipatory anxiety in women 
recalled for futher mammogram breast cancer screening. Psychol Spain 2002; 6:61-7.

9. Benedict $\mathrm{S}$, Williams RD, Baron PL. Recalled anxiety: From discovery to diagnosis of a benign breast mass. Oncol Nurs Forum 1994;4:1723-7.

10. Northouse LL, Jeffs M, Cracchiolo-Caraway A, Lampman L, Dorris G. Emotional distress reported by women and husbands prior to a breast biopsy. Nurs Res1995; 44:196-201. Doi: 10.1097/00006199199507000-00002

11. Seckel MM, Birney MH. Social support, stress, and age in women undergoing breast biopsies. Clinical Nurse Specialist1996; 10: 37-143. Doi: 10.1097/00002800199605000-00009

12. Miles A, Waller J, Wardle J. (2008). Psychological consequences of cancer screening. En: Miller SM, Bowen DJ, Croyle RT, Rowland JH, editors, Handbook of cancer control and behavioral science: A resource for researches, practitioners, and policymakers. Washington: American Psychological Association.p.279-301

13. Absetz P. Determinants and psychological implications of breast cancer risk perceptions in the course of mammography screening. Publications of the Nacional Public Health Institute. 2002. En línea. [Acceso el 7 de junio de 2010]. Disponible en: http:ethesis.helsinki.fi/julkaisut/hum/ psyko/vk/absetz.pdf

14. Wright C. Breast cancer screening: A different look at the evidence. Surgery 1986;100:594-8.

15. Warren R. The debate over mass mammography in Britain: The case for. BMJ 1988; 297:969-70. Doi: 10.1136/ bmj.297.6654.969

16. Marteau TM. Psychological costs of screening. BMJ 1989; 299: 527.

17. Turnbull D, Adelson P, Irwig L. Evaluating the impact of a promotional campaign for screening mammography: Women's knowledge and sources of awareness. Aust J Public Health 1992;16:72-8. Doi:10.1111/j.1753-6405.1992.tb00028.x
18. Steggles S, Lightfoot N, Sellick SM. Psychological distress associated with organized breast cancer screening. Cancer Prev Control 1998;2:213-20.

19. Gram IT, Lund E, Slenker SE. Quality of life following a false positive mammogram. $\mathrm{Br}$ J Cancer 1990;62:1018-22. Doi:10.1038/ bjc. 1990.430

20. Schwartz LM, Woloshin S, Fowler FJJ, Welch HG. Enthusiasm for cancer screening in the United States. JAMA 2004; 291:71-8. Doi:10.1001/jama.291.1.71

21. Brett J, Austoker J. Women who are recalled for further investigation for breast screening: psychological consecuences 3 years after recall and factors affecting re-attendance. J Public Health Med 2001; 23:292-300. Doi: 10.1093/pubmed/23.4.292

22. Brett J, Austoker J, Ong G. Do women who undergo further investigation for breast screening suffer adverse psychological consequences?A multi-centre follow-up study comparing different breast screening result groups five months after their last breast screening appointment. J Public Health Med 1998; 20:396-403. Doi: 10.1093/oxfordjournals.pubmed.a024793

23. Meystre-Agustoni G, Paccaud F, Jeannin A, Dubois-Arber F. Anxiety in a cohort of Swiss women participing in a mammographic screening programme. J Med Screen 2001; 8:213-9. Doi:10.1136/jms.8.4.213

24. Ekeberg Ø, Skjauff H, Kåresen R. Screening for breast cancer is associated with a low degree of psychological distress. Breast 2001;10:20-4. Doi:10.1054/ brst.2000.0177

25. Bull AR, Campbell MJ. Assessment of the psychological impact of breast screening programme. Br J Radiol 1991;64:510-5. Doi:10.1259/0007-1285-64-762-510

26. Scaf-Klomp W., Sanderman R, van de Wiel HB, Otter R., van den Heuvel WJ. Distressed or relieved? Psychological side effects of breast cancer screening in the Netherlands. J Epidemiol Community Health 1997;51:70510. Doi:10.1136/jech.51.6.705 
27. Lampic C, Thurfjell E, Bergh J, Sjödén PO. Short and long term anxiety and depression in women recalled after breast cancer screening. Eur J Cancer 2001;37:463-9. Doi:10.1016/S0959-8049(00)00426-3

28. Espasa R. Impacto psicológico de un falso positivo a la mamografía en un grupo de mujeres participantes en un programa de cribado poblacional de cáncer de mama [tesis doctoral]. Barcelona: Universidad Autónoma de Barcelona. 2009.

29. Manna G, Foddai E, Di Maggio MG, Pace F, Colucci G, Gebbia N, Russo, A. Emotional expression and coping style in female breast cancer. Ann Oncol 2007;18:77-80. Doi: 10.1093/annonc/mdm231

30. Miller S. Monitoring versus blunting styles of coping with cancer influence the information patients want and need about their disease: Implications for cancer screening and management. Cancer 1995;76:167-77. Doi:10.1002/1097$0142(19950715) 76: 2 \% 3$ C167::AIDCNCR2820760203\%3E3.0.CO;2-K

31. Miller S, Champion V. Attitudes about breast cancer and mammography: Racial, income, and educational differences. Women Health 1997; 26:41-63.

32. Miller S, Fang C, Diefenbach M, Bales C. Tailoring psychosocial interventions to the individual's health information-processing style: The influence of monitoring versus blunting in cancer risk and disease. In: Baum A, Anderson $\mathrm{BL}$, editors. Psychosocial interventions for cancer. Washington, DC: American Psychological Association, 2001. p.343-62.

33. Chen CC, David A, Thompson K, Smith C, Lea S, Fahy T. Coping strategies and psychiatric morbidity in women attending breast assessment clinics. J Psychosom Res 1996; 40:265-70. Doi:10.1016/00223999(95)00529-3

34. Clutton S, Pakenham KI, Buckley B. Predictors of emotional well-being following a "false-positive" breast cancer screening result. Psychol Health 1999;14:263-75. Doi: 10.1080/08870449908407327
35. Heckman BD, Fisher EB, Monsees B, Merbaum M, Ristvedt S, Bishop C. Coping and anxiety in women recalled for additional diagnostic procedures following an abnormal screening mammogram. Health Psychol 2004;23:42-

8. Doi:10.1037/0278-6133.23.1.42

36. Pieterse K, van Dooren S, Seynaeve C, Bartels CC, Rijnsburger AJ, de Koning HJ, et al. Passive coping and psychological distress in women adhering to regular breast cancer surveillance. Psychooncology 2007;16: 851-8. Doi:10.1002/pon.1135

37. Mehlsen $M$, Jensen $A B$, Christensen $S$, Pedersen CG, Lassesen B, Zachariae R. A prospective study of age differences in consecuences of emotional control in women referred to clinical mammography. Psychol Aging 2009;24:363-72. Doi: 10.1037/a0015188

38. Cardenal MC, Cruzado JA, González A, Rodríguez A, González MJ, Valentín V. Impacto psicológico de la mamografía de cribado en mujeres con resultados no concluyentes. Progresos Obst Ginecol 2013; 56:393-99. 10.1016/j.pog.2012.10.007

39. Zigmond AS, Snaith PP. The Hospital Anxiety and Depression Scale. Acta Psychiatr Scand 1983; 67:361-70. Doi:10.1111/j.1600-0447.1983.tb09716.x

40. Tejero A, Guimera E, Farre JM, Peri JM. Uso clínico del HAD (Hospital Anxiety and Depression Scale) en población psiquiátrica: un estudio de su sensibilidad, fiabilidad y validez. Rev Psiquiatría Fac Med Barna 1986; 13:233-8.

41. Badía, X, Alonso J. La medida de la salud. Guía de escalas de medición en español. Barcelona: Tecnología y Ediciones del Conocimiento (EDITTEC), 2007.p.60

42. Carver CS, Scheier MF, WeintraubJK. Assesing coping strategies: A theoretically based approach. J Pers Soc Psicol 1989;56:267-83. Doi:10.1037/0022-3514.56.2.267

43. Muñoz M, Roa A, Pérez E, Santos-Olmo AB, de Vicente A. Instrumentos de Evaluación en Salud Mental. Madrid: Pirámide; 2002. p.49. 
